\title{
Spin-drift transport and its applications
}

\author{
Ivar Martin \\ Theoretical Division, Los Alamos National Laboratory, Los Alamos, NM 87545
}

(January 25, 2002)

\begin{abstract}
We study the generation of non-equilibrium spin currents in systems with spatially-inhomogeneous magnetic potentials. For sufficiently high current densities, the spin polarization can be transported over distances significantly exceeding the intrinsic spin-diffusion length. This enables applications that are impossible within the conventional spin-diffusion regime. Specifically, we propose dc measurement schemes for the carrier spin relaxation times, $T_{1}$ and $T_{2}$, as well as demonstrate the possibility of spin species separation by driving current through a region with an inhomogeneous magnetic potential.
\end{abstract}

The rapidly developing field of spin-sensitive electronics promises to reinvigorate conventional semiconductor electronics [1]. Utilizing the carriers' spin degrees of freedom in addition to their charge opens possibilities for conceptually new spintronic devices. Already implemented are GMR read-heads and non-volatile magnetic RAM (MRAM); there are also many proposals for other devices 2. Much of the promise of spintronic devices lies in the enhanced flexibility of the spin degrees of freedom compared to electrical charge. While creating local charge imbalance generally involves large electrostatic Coulomb energies, non-equilibrium spin polarizations can be created at a very low cost. As a consequence, spin polarizations are inherently susceptible to manipulation, e.g. by external magnetic fields or by current drive. Indeed, it has been observed experimentally that nonequilibrium spin polarization can be transported over distances exceeding 100 micrometers [3], with only moderate diffusive spread. This would be impossible in the case of the non-equilibrium charge inhomogeneity, since the Coulomb repulsion would blow such inhomogeneity apart almost instantly.

The immunity of the spin polarization with respect to electrical screening makes it possible to satisfy drift transport conditions, for which the local spin polarization can be carried downstream by an electrical current over distances proportional to the drift velocity. This situation can be obtained for sufficiently large current densities, when the spin-drift length becomes larger than the spin-diffusion length (both defined below). In this Letter we explore this new regime, readily achievable in spin transport, but almost never realized in electron-hole transport. It is studied based on general formulation for spin transport using spin drift-diffusion equations.

The spin-drift regime is generally favorable for spintronic purposes as it can provide larger spin-injection depths compared to the spin-diffusive regime. Here we propose two specific applications of the spin-drift regime: (1) dc transport-based measurement of longitudinal $\left(T_{1}\right)$ and transverse $\left(T_{2}\right)$ spin relaxation times; $(2)$ spin species separation by inhomogeneous magnetic fields. To determine the spin relaxation times, we propose to measure the spin polarization decay profile in the presence of constant current; the decay length can then be related either to $T_{1}$ or $T_{2}$, depending on the experimental setup. Unlike conventional pump-probe techniques, there is no need for ultrafast time-resolved detection, and the spin polarization profile can be measured either by optical [3] or some other techniques. To spatially separate spinup from spin-down carriers, we propose to drive current in a $2 \mathrm{D}$ geometry containing a region of inhomogeneous magnetic field. This technique can be used as a flexible alternative to the standard spin injection techniques.

To study spin transport we use the drift-diffusion approximation for spin currents in the presence of an inhomogeneous spin-dependent potential $V_{\sigma}(\mathbf{r})=-g \mu_{B} \sigma \cdot \mathbf{H}$. At this point we assume that in the plane of the electron gas, the applied magnetic field $\mathbf{H}$ can vary in value but not in orientation. Then all carriers in the sample can be divided into spin-up $(\sigma=1 / 2)$ and spin-down $(\sigma=-1 / 2)$ species that feel magnetic potentials equal in magnitude but opposite in sign, $V_{\uparrow}(\mathbf{r})=-V_{\downarrow}(\mathbf{r})$. For the external potential smaller than the thermal energy in the non-degenerate case, $V_{\sigma}(\mathbf{r})<k_{B} T$, or less than the Fermi energy in the degenerate case, $V_{\sigma}(\mathbf{r})<E_{F}$, the local carrier density will remain essentially unchanged, both with and without current drive. Hence, in what follows we neglect electrical screening effects since they only modify the results in the order $\left(V_{\sigma} / \max \left(k_{B} T, E_{F}\right)\right)^{2}$. In the drift-diffusion approximation, the spin-polarized current is

$$
\mathbf{j}_{\sigma}=n_{\sigma} \mu\left(q \mathbf{E}-\nabla V_{\sigma}\right)-q D \nabla n_{\sigma}
$$

where $q= \pm e$ is the carrier charge, $n_{\sigma}$ the number density of the spin species $\sigma, D$ the diffusion constant, $\mu$ the mobility, $\mathbf{E}$ the external electric field, and $\nabla$ denotes the gradient operator. We assumed that the transport is unipolar (e.g. n-doped semiconductor), and the diffusion constant is the same for both spin species [4]. We do not include the Lorentz force, which also acts on the moving electrons, since it affects both spin species identically and, moreover, is compensated through the classical Hall effect for a confined sample geometry. In practice, local variation of the magnetic potential can be achieved 
either through the use of micromagnets that can create strongly inhomogeneous external magnetic fields, or by magnetically doping semiconductors, which can produce strong position dependence in the carrier $g$-factor [5].

The dynamically generated non-equilibrium spin polarization relaxes towards its equilibrium value. Under constant drive, the relaxing spins are replenished by the divergence of the spin current. In the relaxation-time approximation,

$$
\begin{aligned}
\nabla \cdot \mathbf{j}_{s} & =-\delta_{\sigma} / T_{1}, \\
\delta_{\sigma} & =n_{\sigma}-\bar{n}_{\sigma}, \\
\bar{n}_{\sigma} & =n_{0} \exp \left(-V_{\sigma} / k_{B} T\right) / 2 .
\end{aligned}
$$

The expression for the equilibrium density, $\bar{n}_{\sigma}$, corresponds to non-degenerate carriers. For degenerate carriers, the Boltzman distribution should be replaced by the Fermi-Dirac distribution. In the non-stationary case, there is an extra time derivative, $\partial \delta_{\sigma} / \partial t$, on the left hand side of the continuity equation. Combining Eqs. (11) and (2), the dc equation for spin density under uniform drive $(\nabla \cdot \mathbf{E}=0)$ is

$$
\mu \mathbf{E} \cdot \nabla\left(\bar{n}_{\sigma}+\delta_{\sigma}\right)-\frac{\mu}{q} \nabla\left(\delta_{\sigma} \nabla V_{\sigma}\right)-D \nabla^{2} \delta_{\sigma}=-\frac{\delta_{\sigma}}{T_{1}}
$$

The first term on the left hand side represents the drag of the spin density by the electric field $\left(\mu \mathbf{E}=v_{d}\right.$ - the drift velocity), the second term, as we will see, is usually unimportant, and the last term represents diffusion. Similar descriptions have been previously considered by several authors [6], however, with the focus on the diffusive regime (neglecting $\mathbf{E} \delta_{\sigma}$ terms). The drift terms have only been invoked in the spatially localized regions, such as depletion layers and Schottky barriers [7]. The primary goal of the present work is to explore the spindrift regime that occurs for larger drives, that is when the spin-drift length, $L_{E}=\mu E T_{1}$, exceeds the spin diffusion length, $L_{D}=\sqrt{D T_{1}}$.

We first consider the one dimensional case with spinpolarized negative half-space. The static equilibrium density is

$$
\bar{n}_{\sigma}-n_{0} / 2=\sigma S \Theta(-x),
$$

where $\Theta$ is the step function equal to unity for positive arguments and zero otherwise; $S$ is the static spin density in the polarized region. The step in the density profile implies a step in the magnetic potential, $V_{\sigma}$. The density modification in the presence of a uniform current drive is described by the $1 \mathrm{D}$ version of Eq. (5). The strongest singularity is caused by the jump in $V_{\sigma}$ at $x=0$ (second term in Eq. (5)), which implies discontinuity in the density, $\delta_{\sigma}$,

$$
\frac{\delta_{\sigma}(+0)-\delta_{\sigma}(-0)}{\delta_{\sigma}(+0)+\delta_{\sigma}(-0)}=\frac{\mu}{2 q D}\left(V_{\sigma}(-0)-V_{\sigma}(+0)\right) .
$$

Within our assumptions about the magnitude of the magnetic potential, $V_{\sigma}<\max \left(k_{B} T, E_{F}\right)$, this correction is small and hence can be neglected. Also, it causes charge imbalance, and hence is screened out for distances exceeding the Coulomb screening length [8]. The solution of the homogeneous equation for $\delta_{\sigma}$ is

$$
\begin{aligned}
\delta_{\sigma}(x) & =A \exp \left(\lambda_{1} x\right)+B \exp \left(\lambda_{2} x\right), \\
\lambda_{1(2)} & =\frac{L_{E}}{2 L_{D}^{2}}\left(1+(-) \sqrt{1+4 L_{D}^{2} / L_{E}^{2}}\right) .
\end{aligned}
$$

Matching the $(x>0)$ and $(x<0)$ solutions at $x=0$ and requiring vanishing $\delta_{\sigma}$ at infinity, the spin density, $s=\delta_{\uparrow}-\delta_{\downarrow}$, for the positive drift velocity $(q E>0)$ is

$$
s=\frac{S}{\sqrt{1+4 L_{D}^{2} / L_{E}^{2}}} \times\left\{\begin{array}{ll}
\exp \left(\lambda_{1} x\right), & x<0 \\
\exp \left(\lambda_{2} x\right), & x>0
\end{array} .\right.
$$

The solution for the negative drift velocity can be obtained from this solution as $-s(-x)$.

Two interesting special cases correspond to the spindrift $\left(L_{E} \gg L_{D}\right)$ and the spin-diffusion $\left(L_{E}<L_{D}\right)$ regimes. In the spin-drift case,

$$
s= \begin{cases}S \exp \left(-x / L_{E}\right), & x>0 \\ 0, & x<0\end{cases}
$$

And in the spin-diffusion case,

$$
s=\frac{S L_{E}}{2 L_{D}} \exp \left(-|x| / L_{D}\right) .
$$

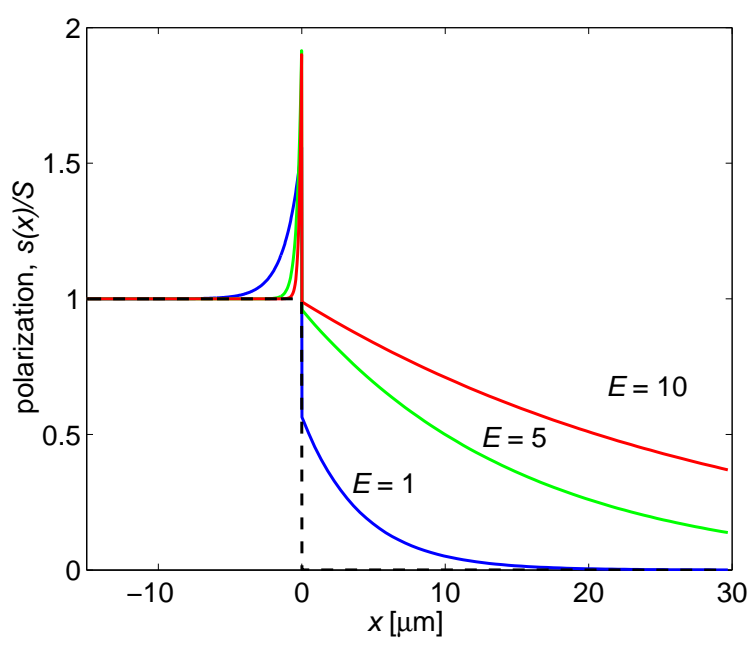

FIG. 1. Spin polarization drag induced by electric current in 1D geometry, Eq. (9). The dashed line corresponds to the spin polarization profile in the absence of current. The indicated strength of the electric field, $E$, is in $\mathrm{V} / \mathrm{cm}$. Note that the stronger the applied electric field is, the farther away the spin polarization can be dragged. The parameters are characteristic of $10^{16} \mathrm{~cm}^{-3}$ Si-doped GaAs: $\mu=3000 \mathrm{~cm}^{2} / \mathrm{Vs}$, $T_{1}=100 \mathrm{~ns}, T=1.6 \mathrm{~K}$. Carriers are assumed to be non-degenerate. 
The series of solutions for various strengths of the electric field are shown in Figure 11. The parameters are characteristic of $10^{16} \mathrm{~cm}^{-3}$ Si-doped GaAs [3]: $\mu=$ $3000 \mathrm{~cm}^{2} / \mathrm{Vs}, T_{1}=100 \mathrm{~ns}, T=1.6 \mathrm{~K}$. The drift condition for this system is realized for $E>2 \sqrt{k_{B} T / q \mu T_{1}} \sim$ $2 \mathrm{~V} / \mathrm{cm}$. Experimentally, much larger electric fields have been applied to this system [3].

Under spin-drift conditions, the characteristic length of the exponential decay of spin polarization is proportional to the longitudinal spin relaxation time, $L_{E}=v_{d} T_{1}$. In this regime, there is almost one-to-one correspondence between the distance from the interface and the time spent by electrons in the region with zero field, $x=v_{d} t$, which is only weakly altered by diffusion. Therefore, by measuring the decay length and knowing the drift velocity, $v_{d}=\mu E$, one can directly determine $T_{1}$. An advantage compared to the conventional pump-probe approach [3] is that the measurement can be conducted in a dc setup that doesn't require ultrafast optics and electronics. The spin polarization detection with sub-micron spatial resolution can be done by standard optical techniques based on Kerr/Faraday rotation, or possibly using magnetic resonance force microscopy (MRFM) [9 by modulating the current at the cantilever frequency [10].

In addition to the longitudinal relaxation time, $T_{1}$, which defines how fast the longitudinal spin polarization disappears, an almost identical approach can be applied to determine the transverse spin relaxation time, $T_{2}$, which measures the electron spin decoherence time. For that, in the setup described above, a weak uniform magnetic field, $H_{\perp}$, should be applied perpendicular to the direction of the strong field that exists at $x<0$. In the region with the strong field, this additional component will only weakly alter the the direction of the total field. However, in the region where originally there was no magnetic field, this component will cause the precession of the drifting spin polarization. The spatial period of the precession

$$
L_{P}=2 \pi v_{d} / \omega_{L}
$$

is related to the Larmor frequency, $\omega_{L}=g \mu_{B} H_{\perp} / \hbar$, which is about $62 \mathrm{MHz}$ at $H_{\perp}=100 \mathrm{G}$ for $g=-0.44$. For the driving electric field $E=10 \mathrm{~V} / \mathrm{cm}$ and the above parameters, $L_{P} \sim 5 \mu \mathrm{m}$. Such spatial modulation can be resolved both by optical means or with MRFM. To determine the transverse spin relaxation time $T_{2}$ one needs to measure spatial dependence of the spin polarization component perpendicular to $H_{\perp}$ and then fit it to

$$
S \propto \exp \left(-x / v_{d} T_{2}\right) \cos \left(2 \pi x / L_{P}\right) .
$$

Here we neglected the diffusive spread of the spin polarization, which will case an analogue of NMR inhomogeneous broadening. The diffusive spread will matter, however, only when it becomes comparable to the precession length, $L_{P}$. This effectively places an upper limit on the distance from the interface for which the precessioninduced oscillation can be resolved, $L<\left|\mu E L_{P}^{2} / D\right| \sim$ $250 \mu \mathrm{m}$ for the above parameters. The upper limit can be modified by the choice of the transverse magnetic field $B_{\perp}$ with the only constraint that $\omega_{L} T_{2}>1$. Finally, the carrier $g$-factor itself can be determined from the Larmor frequency, which can be extracted from the precession length.

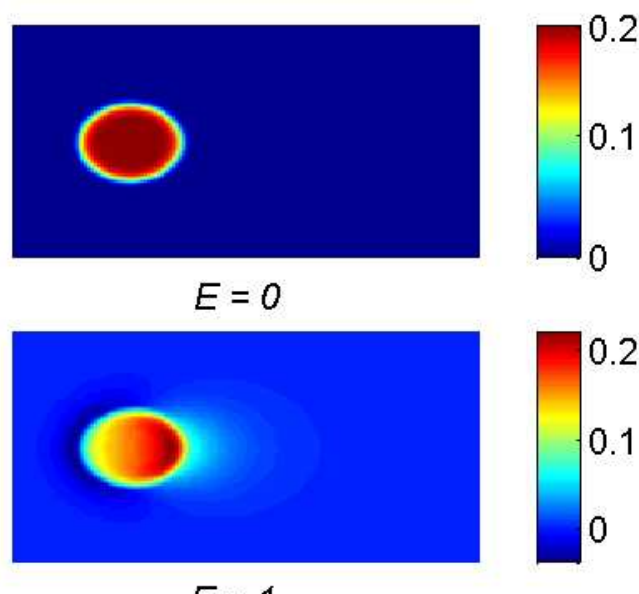

$E=1$

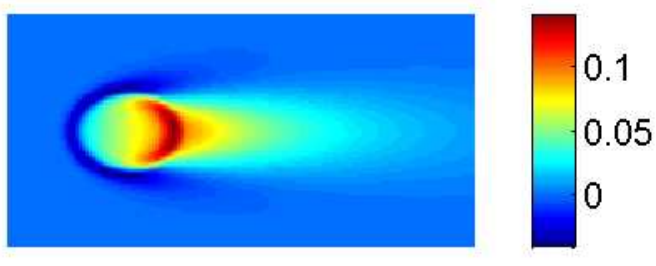

$E=5$

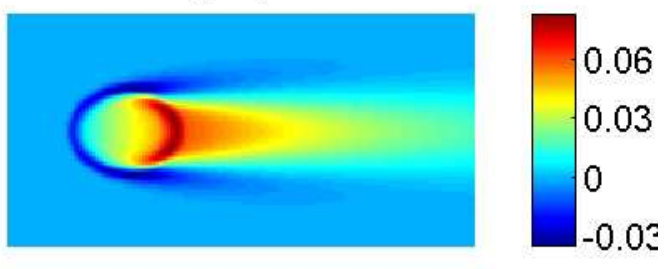

$E=10$

FIG. 2. Spin species separation by inhomogeneous magnetic field. An electric current flows in $2 \mathrm{D}$ (film) through a region of localized magnetic field, $V_{\sigma}(r)=-0.6 \sigma \exp \left[-(r / 5)^{10}\right]$, with $r$ in micrometers and energy $V_{\sigma}$ in Kelvin. The spin polarization is measured in the units of the total density. The strength of the electric field, $E$, is in $\mathrm{V} / \mathrm{cm}$. Notice the region of negative ("minority") spin polarization that first forms were the current impinges on the region of the magnetic field. For large enough drift velocities, the "majority" and the "minority" carriers forms a three-tail structure that extends over the drift length, $L_{E}$, downstream. The material parameters are the same as in Fig. 1.

Another application that is only possible in the spindrift regime is the spatial separation of spin species by driving electric current through a region with an inhomogeneous magnetic field. To demonstrate this effect, we consider a two-dimensional system with a compact region 
containing magnetic field. Unlike the one-dimensional case considered above, the "minority" (opposite to magnetic field) spin carriers can avoid this region by flowing around it. Indeed, this is what we observe by solving numerically the $2 \mathrm{D}$ spin-relaxation drift-diffusion equations, Fig. 2. The calculation is performed on a $100 \times 150$ lattice that spans $30 \times 45 \mu \mathrm{m}$ region for parameters relevant to Si-doped GaAs (specified above). The field is localized in $10 \mu \mathrm{m}$ disk. For large enough electric field, there is formation of the negative spin polarization where the current impinges on the boundary of the magnetic field region [11]. The minority spin polarization is then dragged downstream by the current until it relaxes and diffuses away. Notice that in the zero-current state there is no negative spin polarization in the system. The "majority" spin polarization is ripped off the local field region, and together with the "minority" carriers forms a three-tail structure that extends over the spindrift length, $L_{E}$, downstream. Therefore, driving current through this system leads to an effective separation of the two spin species. A similar effect can be expected also in the ballistic regime realized in the ultra-high mobility 2DEG structures.

The effect that we discussed above is analogous to the classic Stern-Gerlach effect; however, unlike the original effect the spin separation can be achieved even with electrons. In the original Stern-Gerlach experiment, neutral spin- $1 / 2$ particles passing through a region with a magnetic field gradient get deflected by the force $F=$ $g \mu_{B}(\sigma \cdot \nabla) \mathbf{H}$, and produce two spots on the screen that represent two possible projections of spin $\sigma$. For charged particles, the situation is complicated due to the Lorentz force that acts to scramble the separation. It was first pointed out by Bohr that in the case of electrons, the observation of Stern-Gerlach effect is impossible because the deflection by the Lorentz force exceeds the spin-related splitting [12]. The Bohr argument does not apply, however, to constrained geometries, e.g. a thin film sample, where the Lorentz force is compensated by the Hall voltage. This makes the solid-state Stern-Gerlach effect possible. It is important to stress, however, that despite the appearance, the mechanism for spin separation here is distinctly different from the the classic Stern-Gerlach effect. A time-dependent Stern-Gerlach effect was recently considered by Fabian and Das Sarma 13].

In the above discussion we assumed a possibility of a magnetic field profile that is inhomogeneous but always pointing along the same line, e.g. perpendicular to the sample plane. This is an important assumption because the fringe fields have ability to destroy spin polarization through precession. For the $10^{16} \mathrm{Si}$ GaAs, a 100 Gauss fringe field at $10 \mathrm{~V} / \mathrm{cm}$ drive could scramble the polarization after $5 \mu \mathrm{m}$. However, since the fringe fields decay rapidly in space, their detrimental effects can be reduced by increasing the drive or by reducing the size of the micromagnets. Moreover, for thin-film samples, the effects of the fringe fields can be essentially eliminated by placing the sample between two closely spaced magnet ter- minals (split ring) or between two "identical" magnets. Alternatively, one could selectively boost the $g$-factor, e.g. by locally doping II-VI semiconductors with Mn. This way, when placed even in a relatively weak uniform external magnetic field, different parts of the sample will feel very different magnetic potentials. By combining these approaches, one can engineer a variety of strong magnetic potentials which are not affected by the fringe fields.

In summary, we explored the spin-drift regime, which is realized for sufficiently large but realistic current densities in semiconductors. In this regime, the spin density can be carried by the current over distances controlled by the spin-drift length, which can significantly exceed the spin-diffusion length. This opens a possibility of designing spintronic devices with the spin properties dynamically controllable by the applied bias. This is in sharp contrast with the conventional particle-hole semiconductor electronics where electrostatic screening practically precludes large scale dynamic manipulation of the relative particle-hole density. As specific applications, we proposed measurement schemes for the carrier spin relaxation times, $T_{1}$ and $T_{2}$, as well as demonstrated the possibility of spin species separation by driving current through a region with an inhomogeneous magnetic potential. The latter is analogous to the classic Stern-Gerlach effect.

Upon completion of this paper, author became aware of the related one-dimensional study of the spin-drift regime by $\mathrm{Yu}$ and Flatté 14].

We thank S.A. Crooker, J. Albrecht, D.L. Smith, J. Fabian, and S.A. Trugman for useful discussions. This work was supported by DARPA SPINs program.

[1] G. Prinz, Science 282, 1660 (1998); S.A. Wolf et al., Science 294, 1488 (2001).

[2] For an extensive list of references, see I. Žutić, preprint cond-mat/0112368 (2001).

[3] J. Kikkawa and D.D. Awschalom, Nature (London)397, 139 (1999).

[4] For detailed analysis of spin diffusion in doped and intrinsic semiconductors, see M.E. Flatte and J.M. Buyers, Phys. Rev. Lett. 84, 4220 (2000).

[5] Y. Ohno et al., Nature (London) 402, 790 (1999); M. Oestreich et al., Appl. Phys. Lett. 74, 1251 (1999); R. Freiderling et al., Nature (London) 402, 787 (1999); B.T. Jonker et al., Phys. Rev. B 62, 8180 (2000).

[6] Mark Johnson and R. H. Silsbee, Phys. Rev. B 35, 4959 (1987); D.L. Smith and R.N. Silver, Phys. Rev. B 64, 5323 (2001).

[7] Žutić, J. Fabian, and S. Das Sarma, preprint condmat/0106085 (2001); J. Albrecht and D.L. Smith, unpublished.

[8] H.U. Baranger and J.W. Wilkins, Phys. Rev. B 30, 7349 (1984); S.A. Trugman and A.J. Taylor, Phys. Rev. B 33, 5575 (1986). 
[9] J. A. Sidles , Phys. Rev. Lett. 68, 1124 (1992); D. Rugar et al., Science 264, 1560 (1994); Z. Zhang, P. C. Hammel, and P. E. Wigen, Appl. Phys. Lett. 68, 2005 (1996);

[10] M. Roukes and P.C. Hammel, private communication.

[11] In the $1 \mathrm{D}$ geometry, the dynamical generation of the "negative" polarization has been observed by Žutić, J. Fabian, and S. Das Sarma, preprint cond-mat/0106085 (2001).
[12] For a recent discussion of the Bohr-Pauli-Mott argument and possible exceptions, see B. M. Garraway and S. Stenholm, Phys. Rev. A 60, 63 (1999).

[13] J. Fabian and S. Das Sarma, preprint cond-mat/0104146 (2001).

[14] Z. G. Yu and M. E. Flatté, preprint cond-mat/0201425 (2002). 